\title{
An ATP-EMTP-Based Model for Analysis of Shielding Properties of Ferromagnetic Cable Sheaths
}

\author{
Marjan Popov, Senior Member, IEEE, Leonid Grcev, Senior Member, IEEE, Lou van der Sluis, Senior Member, IEEE, \\ and Vladimir V. Terzija, Senior Member, IEEE
}

\begin{abstract}
Ferromagnetic materials, such as steel, are used for shielding of power and telecommunication cables. These shields often provide path for currents during faults or lightning and are important for the integrity and safety of electrical systems and devices. Since such currents might be with low- or high-frequency content, and of low or high intensity, ferromagnetic shield behavior might be quite different due to hysteresis. This paper describes a method for analyzing the dynamic behavior of ferromagnetic cable shields based on the Jiles model of hysteresis. The solution is implemented in ATP-EMTP, which allows one to analyze the complex behavior of ferromagnetic cable shields in conjunction with that of the other elements of the power system. Due to limitations of the model of hysteresis, application of the proposed model is limited to frequencies up to about $10 \mathrm{kHz}$. The model is illustrated for a practical 100-m-long cable.
\end{abstract}

Index Terms-ATP-EMTP, cable, hysteresis, Jiles model, magnetic field, MODELS.

\section{INTRODUCTION}

$\mathbf{T}$ ODAY, the problem of hysteresis is well understood and several mathematical models have been developed for simulating the dynamic behavior of ferromagnetic materials [1]. Two of these are often used in studies on transients in electrical machines and in other devices with magnetic materials, which describe the hysteresis, the Preisach model, [2] and the Jiles model [2]-[4].

Since the analysis of power and telecommunications networks is based on circuits, it is necessary to derive the correlation between currents and voltages and to determine the parameters of equivalent circuits. In the case where ferromagnetics are involved, as with transformer cores or cable sheaths, the inductances are nonlinear and the magnetic permeability changes in every particle of the ferromagnetic material. Often, hysteresis must be taken into account, as the losses of the material or shielding properties can be significantly influenced by current frequency and magnitude. Such analysis requires knowledge of the magnetic field and fluxes in the material.

Manuscript received October 28, 2003; revised August 20, 2004. Paper no. TPWRD-00544-2003.

M. Popov and L. van der Sluis are with the Power Systems Laboratory, Delft University of Technology, Delft 2628 CD, The Netherlands (e-mail: M.Popov@ieee.org; L.vanderSluis@EWI.TUDelft.NL).

L. Grcev was with the Department of Electrical Engineering, Eindhoven University of Technology, Eindhoven $5600 \mathrm{MB}$, The Netherlands. He is now with the Faculty of Electrical Engineering, Saints Cyril and Methodius University, Skopje 1000, Macedonia (e-mail: leonid.grcev@ieee.org).

V. V. Terzija is with ABB Calor Emag Mittelspannung GmbH, Ratingen 40472, Germany (e-mail: Terzija@ieee.org).

Digital Object Identifier 10.1109/TPWRD.2004.843398
A detailed analysis of the dynamic current distribution in the sheath is of primary interest when one studies currents induced in the central conductors by currents impressed on the shield; for example, due to lightning or fault [5]. Looking for a more complete approach to modeling, the following phenomena were considered important:

- current distribution in the shield due to skin effect;

- surge propagation along the cable;

- interactions between the cable and other parts of the power system;

- coupling between the cable sheaths and the grounding system, especially near the bonding points.

In relation to the first point above, the current distribution in the shield has been analyzed in [6] for a simplified saturable ferromagnetic model and in [7] for a full Jiles hysteresis model. However, in these works ([6], [7]), propagation along the cable is ignored.

Surge propagation along the cables is traditionally modeled using transmission-line theory, for example, [8]-[10]. The effects of surge propagation in cable shields with magnetic saturation are analyzed in [11], but hysteresis was neglected. The equivalent circuit approach with linear inductances is presented in [12] and [13].

One of the most efficient ways to model cables together with the power system is to use ATP-EMTP [14]. Standard ATPEMTP cable models [14] can be used to simulate the transients in the phase conductors. However, for the transients along the sheath, a user-defined model is needed, which may be facilitated using ATP's MODELS. A detailed EMTP cable model that simulates transients along the sheath is described in [15]. However, this model uses linear inductances and is applicable for sheaths with weak magnetic properties.

Recent experimental analysis has confirmed that ferromagnetic shields might have a large influence on the distribution of the fault current between the substation grounding systems and cable shields at low frequencies [16]. A similar conclusion was also drawn from a simulation study in [17] for frequency ranges up to $10 \mathrm{kHz}$.

This paper presents an attempt for more complete modeling that includes a dynamic model of hysteresis. The equivalent dynamic permeability is determined at any point in the shield [7], from the electromagnetic (EM) fields solved in time domain in the shield, taking into account the ferromagnetic hysteresis using the Jiles dynamic model [4]. The equivalent permeability is further used for inductances in an equivalent circuit of the cable shield [8], [9]. This enables an analysis of the propagation along cable shields. The whole solution is implemented within ATP-MODELS. The set of partial differential equations 
(PDE's) for the fields in time domain is solved by a finite difference scheme in ATP's MODELS. The Jiles equation is solved simultaneously and for each finite element, a different permeability is updated into the PDEs. This results in an equivalent black-box circuit that is interfaced into ATP and can also be used for more complicated cable models.

An application example of the full model is also presented, involving transient wave propagation along the sheath of a $100-\mathrm{m}-$ long cable.

It should be noted that the dynamic Jiles model of hysteresis has been applied so far for frequencies up to about $10 \mathrm{kHz}$ [21], which should be considered also as a limitation of this model.

\section{Outline OF the BAsic Steps In the SOlution}

The solution is based on an equivalent circuit of the cable sheath. For this purpose, the cable of length $l$ is segmented in a number of longitudinal segments $n$, each represented by an equivalent circuit. For each longitudinal segment, uniform parameters and current and voltage distributions are assumed. A rule of thumb is that the length of such segments $l / n$ should be a fraction of the wavelength $\lambda$ in the medium for the highest frequency considered (for example, $l / n=\lambda / 10$ [18]). It is worth noting that in the soil, $\lambda$ might be much shorter than in the air [18].

The next step is to model the skin effect for a dynamic excitation. For that purpose, each segment is divided in layers assuming uniform parameters in the layer. Each layer and the mutual influences of the layers are represented with equivalent circuit representations. This step is repeated for each longitudinal segment for the total current in the segment depending on the propagation along the cable.

The internal impedance is therefore determined by solving Maxwell's equations subjected to boundary conditions in the time domain and taking into account nonlinear hysteresis phenomena. However, the external impedance follows from a discretized approximation of the usual transmission-line solution of buried horizontal conductors [9].

Presently, the mutual interactions between the cable and the elements of the grounding system have not been implemented in the model. However, future work might follow the solutions suggested in [22].

\section{Equivalent Circuit of The CABle Sheath}

\section{A. Equivalent Circuit of One Cable Segment}

Fig. 1 illustrates the equivalent circuit of one cable segment whose sheath is divided in $J-1$ layers used for modeling the skin effect in time domain.

Here, mutual inductances between layers $L_{j-1, j}$ and resistances $R_{j}$ are calculated according to [12] and [13]

$$
\begin{aligned}
L_{j-1, j} & =\frac{\mu_{D} \Delta l}{2 \pi} \cdot \ln \frac{d_{j}-\Delta r}{d_{j}-3 \Delta r} \\
R_{j} & =\frac{\rho \Delta l}{\pi \cdot \Delta r \cdot\left(d_{j}-\Delta r\right)}
\end{aligned}
$$

where $d_{j}$ is the outer diameter of the $j$ th layer of the sheath, $\Delta r$ is thickness of the layers, and $\Delta l=l / n$ is the length of the cable segment, where $l$ is the length of the cable and $n$ the number of segments.

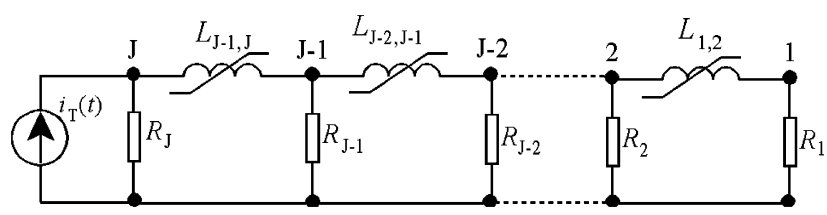

Fig. 1. Equivalent circuit of a cable sheath divided into $J-1$ layers for one cable segment.

This equivalent circuit is well known [12], [13] and is experimentally verified in [24]. In this paper, we introduce nonlinear inductances that represent the hysteresis in each layer. In (1), $\mu_{D}$ is the dynamic permeability that in our case is nonlinear.

The equivalent circuit in Fig. 1 completes the model of the internal impedance elements of the sheath. What is left is the modeling of external impedance elements due to surrounding earth.

\section{B. Complete Equivalent Circuit of a Cable Sheath}

A model of a complete cable (Fig. 2) consists of serial connections of short longitudinal proportional-integral (PI) segments, where each segment is represented by the equivalent circuit in Fig. 1 [12], [13]. The corresponding equivalent circuit of a cable sheath with a length $l$ is shown in Fig. 2, where $C_{t}, R_{t}$, and $L_{t}$ are the capacitance, resistance, and inductance to the earth, respectively [9]

$$
\begin{aligned}
C_{t} & =\frac{l}{n} \cdot \frac{\pi \varepsilon_{0} \varepsilon_{r}}{\ln \frac{2 l}{\sqrt{d_{a} h}}-1} \\
R_{t} & =\frac{n}{l} \cdot \frac{\rho_{E}}{\pi}\left(\ln \frac{2 l}{\sqrt{d_{a} h}}-1\right) \\
L_{t} & =\frac{l}{n} \frac{\mu_{0}}{2 \pi}\left(\ln \frac{2 l}{\sqrt{d_{a} h}}-1\right) .
\end{aligned}
$$

In the above equations, $d_{a}$ is the outer diameter of the conductor in $(m)$, and $h$ is the deepness of the buried cable in $(m) ; \rho_{E}$ and $\epsilon_{r}$ are the specific resistance in $(\Omega \mathrm{m})$, and the relative permittivity of the earth, respectively.

It is worth mentioning that although (3)-(5) are approximate, they have proven to be applicable for modeling earthing effects for buried conductors segmented in short segments in frequency ranges up to $1 \mathrm{MHz}$ in comparison with experimental results [23].

\section{EVAluation OF THE DyNAMIC PERMEABILITY}

\section{A. Time-Domain Fields in the Sheath}

For simplicity, we consider a long thin tubular cable with an outer sheath of ferromagnetic material and a single concentric central conductor as shown in Fig. 3. This configuration is considered also for validation purposes to compare results with the model [6] (Section VI). The details of the development in this section are available elsewhere [6], [7]; here, only the basic steps of the solution are included for completeness. The total sheath current $i_{T}(t)$ is impressed uniformly along the cable. The shielding effectiveness is estimated using the relation between the current induced in the center of the conductor $i_{C}(t)$ by the impressed current $i_{T}(t)$. 


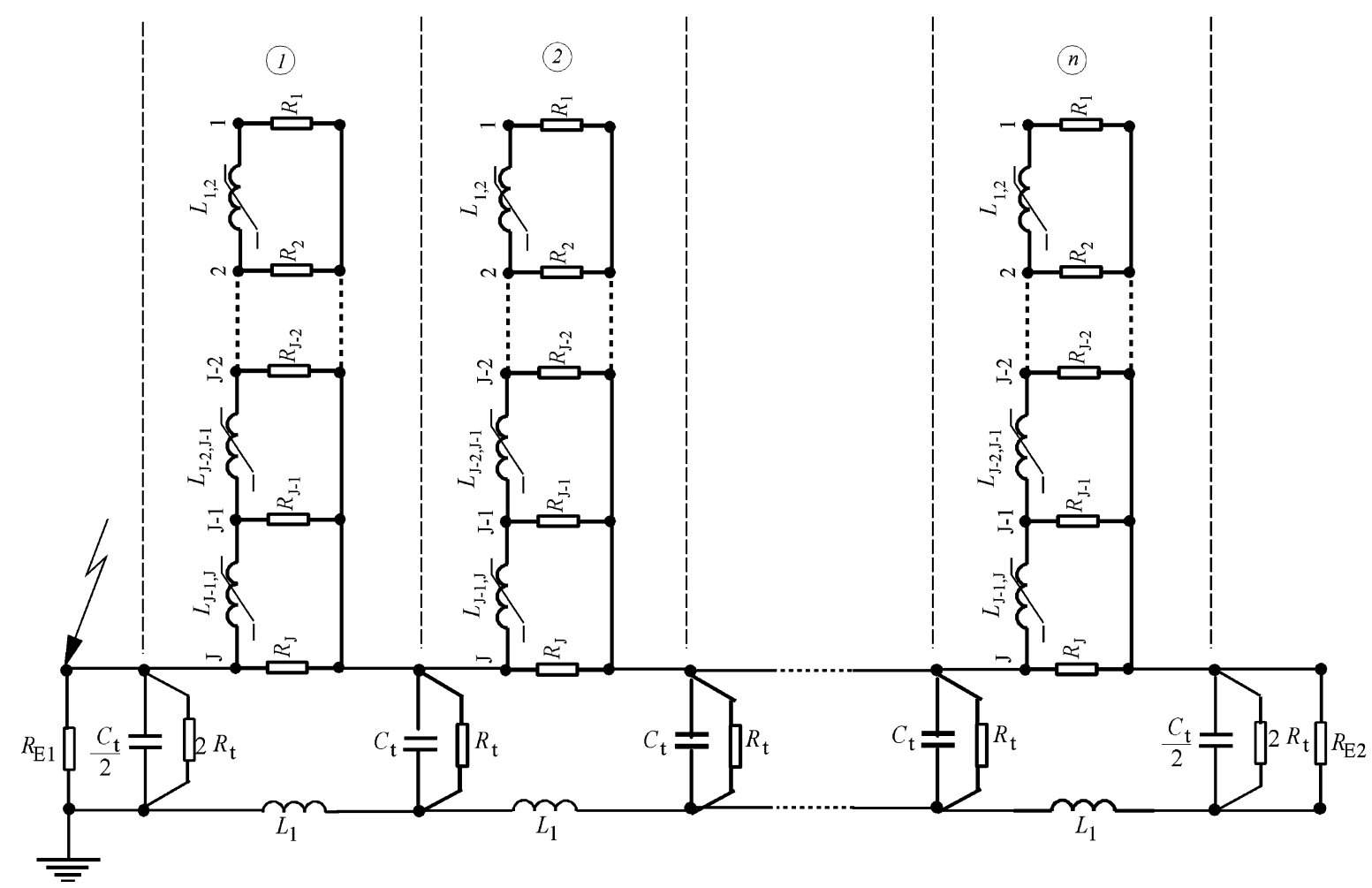

Fig. 2. Complete equivalent circuit of a cable sheath considering continuous current flow in the earth.

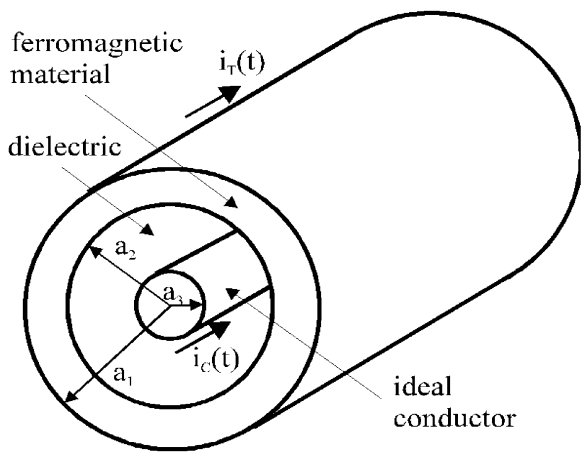

Fig. 3. Tubular ferromagnetic shield with a single concentric conductor.

Field components in the outer sheath and the separating dielectric satisfy [6], [7]

$$
\frac{\partial E_{z}}{\partial r}=\frac{\partial B_{\phi}}{\partial t} \text { and } \frac{1}{r}\left[\frac{\partial\left(r H_{\phi}\right)}{\partial r}\right]=\sigma E_{z}+\varepsilon_{0} \frac{\partial E_{z}}{\partial t}
$$

where the $r$-, $z$-, and $\phi$-axis represent the cylindrical coordinate system, and $\varepsilon_{0}\left(\partial E_{z} / \partial t\right)$ is a displacement current that for simplicity will be neglected henceforth. The components of the magnetic and electric field in the separating dielectric are

$$
H_{\phi}=\frac{i_{C}(t)}{2 \pi r} \text { and } E_{z}=\frac{\mu_{0}}{2 \pi} \frac{\partial i_{C}(t)}{\partial t} \ln \frac{r}{a_{3}} .
$$

In the ferromagnetic sheath, (1) can be written as

$$
\frac{\partial^{2} H_{\phi}}{\partial r^{2}}+\frac{1}{r} \frac{\partial H_{\phi}}{\partial r}-\frac{H_{\phi}}{r^{2}}=\sigma \frac{\partial B_{\phi}}{\partial H_{\phi}} \frac{\partial H_{\phi}}{\partial t} .
$$

The boundary conditions for $E_{z}$ and $H_{\phi}$ at the inner surface of the sheath can be combined by (6) and (7) into one equation with a boundary condition

$$
\frac{H_{\phi}}{a_{2}}+\frac{\partial H_{\phi}}{\partial r}=\left.\sigma \mu_{0} a_{2} \frac{\partial H_{\phi}}{\partial t} \ln \frac{a_{2}}{a_{3}}\right|_{r=a_{2}} .
$$

The total shield current $i_{T}(t)$ is related to the magnetic field intensity at the outer shield boundary as

$$
\left.H_{\phi}\right|_{r=a_{1}}=\frac{i_{T}(t)}{2 \pi a_{1}} .
$$

It has to be pointed out that the central conductor is considered as not loaded and the only current that the central conductor carries $i_{C}(t)$ is the current induced from the impressed current on the sheath surface $i_{T}(t)$. By determining the field distribution in layers inside the sheath, it is possible to determine the induced current in the conductors in the sheath. For example, the current induced in the central conductor in Fig. 3 is determined by the field in the inner sheath layer $i_{C}(t)=\left.2 \pi a_{2} H_{\phi}\right|_{r=a_{2}}$.

It is necessary to determine the dynamic permeability $\mu_{D}=$ $\partial B_{\phi} / \partial H_{\phi}$ in (8) in order to complete the solution for which we use the following model of ferromagnetic hysteresis.

\section{B. Model of a Ferromagnetic Hysteresis}

The following form of the Jiles model [4] is used:

$$
\frac{d M}{d H}=\frac{M_{\mathrm{an}}-M+k \cdot \delta \cdot c \cdot \frac{d M_{\mathrm{an}}}{d H_{e}}}{k \cdot \delta-a \cdot\left(M_{\mathrm{an}}-M+k \cdot \delta \cdot c \cdot \frac{d M_{\mathrm{an}}}{d H_{e}}\right)} .
$$

The Jiles model is based on five parameters that depend on the used material:

$M_{s}$ magnetic saturation in $\mathrm{A} / \mathrm{m}$;

$\alpha \quad$ mean field parameter; 
a shape parameter in $\mathrm{A} / \mathrm{m}$;

$k$ domain wall pinning constant;

$c$ domain wall flexing constant.

Here, $\delta$ is given by

$$
\delta= \begin{cases}1, & \text { if } \frac{d H}{d t}>0 \\ 0, & \text { otherwise }\end{cases}
$$

The anhysteretic magnetization function $M_{\mathrm{an}}$ is given by

$$
M_{\mathrm{an}}=M_{s} \cdot\left[\operatorname{coth}\left(\frac{H_{e}}{a}\right)-\frac{a}{H_{e}}\right]
$$

and the effective field $H_{e}$ in (11) is calculated by

$$
H_{e}=H+\alpha M
$$

The solution of (11) for $d M / d H$ leads to $d B / d H$ required in (8), which completes the formulation.

\section{Numerical Solution}

For the integration of (8), a finite-differencing scheme is used, and the rectangular grid of points is defined in the $r$ - $t$ plane. The derivatives in (8) and (9) are substituted by finite-difference approximations. The backward differencing representation of the time-derivative terms and a center differencing representation of the space derivative terms is chosen, that is

$$
\begin{aligned}
\frac{\partial h}{\partial t} & =\frac{h_{j}^{k+1}-h_{j}^{k}}{\Delta t}, \quad \frac{\partial h}{\partial r}=\frac{h_{j+1}^{k+1}-h_{j-1}^{k+1}}{2 \Delta r} \\
\frac{\partial^{2} h}{\partial r^{2}} & =\frac{h_{j+1}^{k+1}-2 h_{j}^{k+1}+h_{j-1}^{k+1}}{\Delta r^{2}}
\end{aligned}
$$

where $\Delta r=\left(a_{1}-a_{2} / J-1\right), J$ is the number of points between $r=a_{1}$ and $r=a_{2}$, and $\Delta t$ is the time increment. In this way, recursive expressions can be derived that can be implemented simply in MODELS. This is important as it saves computation time in ATP. Substituting (14) in (8) and (9) gives

$$
\begin{aligned}
& \frac{h_{j+1}^{k+1}-2 h_{j}^{k+1}+h_{j-1}^{k+1}}{\Delta r^{2}}+\frac{1}{r_{j}} \frac{h_{j+1}^{k+1}-h_{j-1}^{k+1}}{2 \Delta r}-\frac{h_{j}^{k+1}}{r_{j}^{2}} \\
& =\sigma \mu \frac{h_{j}^{k+1}-h_{j}^{k}}{\Delta t} \\
& j=2, \ldots, J-1 \\
& \frac{h_{1}^{k+1}}{a_{2}}-\frac{3 h_{1}^{k+1}-4 h_{2}^{k+1}+h_{3}^{k+1}}{2 \Delta r} \\
& =\sigma \mu_{0} a_{2} \ln \left(\frac{a_{2}}{a_{3}}\right) \frac{h_{1}^{k+1}-h_{1}^{k}}{\Delta t} \\
& h_{J}^{k+1}=\frac{1}{2 \pi a_{1}} i_{T}\left(t_{k+1}\right) .
\end{aligned}
$$

The value of the dynamic permeability

$$
\mu_{D}=\frac{\partial B}{\partial H}
$$

is obtained by solving (11) by a fourth-order Runge-Kutta method, and it is different for each layer. Equation (15) consists of a set of $J$ nonlinear equations that are solved for every time step.

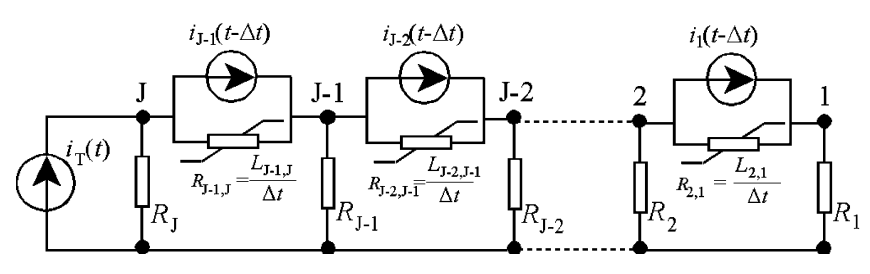

Fig. 4. Modified equivalent circuit for layers in one segment.

TABLE I

PARAMETERS OF THE JILES MODEL

\begin{tabular}{l|c|c|c|r}
\hline \multicolumn{5}{c}{ Hysteresis characteristic } \\
\hline$M_{s}(\mathrm{~A} / \mathrm{m})$ & $a(\mathrm{~A} / \mathrm{m})$ & $k(\mathrm{~A} / \mathrm{m})$ & $\alpha$ & $c$ \\
\hline $1,42 \times 10^{6}$ & 55 & 120 & $1 \times 10^{-6}$ & 1 \\
\hline
\end{tabular}

TABLE II

PARAMETERS FOR THE CABLE SHEATH

\begin{tabular}{c|c|c|c}
\hline$\sigma(\mathrm{mho} / \mathrm{m})$ & $a_{1}(\mathrm{~m})$ & $a_{2}(\mathrm{~m})$ & $a_{3}(\mathrm{~m})$ \\
\hline $10^{7}$ & $6.35 \times 10^{-3}$ & $6.223 \times 10^{-3}$ & $2.7045 \times 10^{-3}$ \\
\hline
\end{tabular}

\section{IMPLEMENTATION OF THE MODEL IN ATP-EMTP}

The equivalent circuit in Fig. 2 is implemented in ATP-EMTP. The set of (15) together with (11) is solved for each time step by MODELS interfaced to ATP-EMTP, from which the dynamic permeability is determined. Each nonlinear inductance is represented by a parallel connection of a current source (type-60) and a variable resistance (type-91), as shown in Fig. 4.

The source $i_{T}$ is also a MODELS-controlled source, and in each time step, it is exported by a type-60 element into ATPEMTP. In this way, the skin effect can be easily observed for different types of current excitations. The current source type-60 is defined by its amplitude and direction. It should be pointed out that each current source representing the nonlinear inductance is formed by two type-60 sources; one with a negative value of the current $i_{j-1}(t-\Delta t)$, and the other with a positive value of the same current. In this way, one can provide a current source between two nodes.

The current sources $i_{j-1}(t-\Delta t)$ take into account the history of the current in the inductive element from the preceding time step [14].

\section{NUMERICAL RESULTS}

\section{A. Long Coaxial Cable}

To compare results from the model with previously published results, the described procedure is applied to a long thin coaxial cable [6]. The parameters of the Jiles model for a very thin hysteresis curve that are compatible with the saturable model in [6] are summarized in Table I. The procedure of how to obtain the real data for a specific magnetic material is explained in [4]. Another very useful technique for determining Jiles parameters from known hysteresis loops is presented in [20]. Cable data are shown in Table II. The number of layers on which the cross section is divided is 19 . 

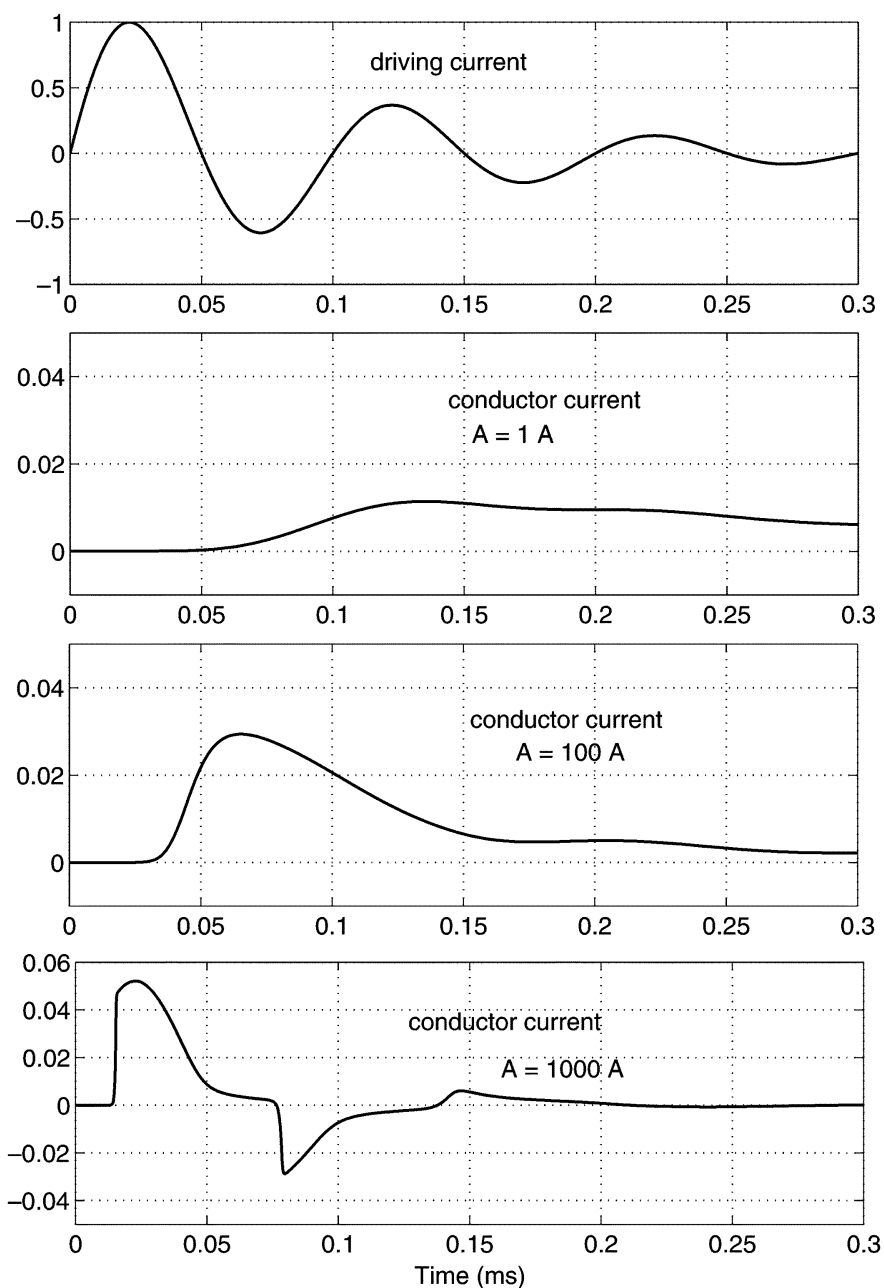

Fig. 5. Normalized center conductor current for a damped sine wave of the driving current $i_{T} ; f_{0}=10 \mathrm{kHz}$ applying the ATP-EMTP model (thin hysteretic characteristic).

The driving current is taken to be a damped sinusoid with a frequency of $f_{0}=10 \mathrm{kHz}$

$$
i_{T}=1.2678 \cdot A \cdot e^{-f_{0} t} \cdot \sin 2 \pi f_{0} t
$$

where $A$ is the amplitude of the applied current. The conductor current is computed by applying the driving current $i_{T}$ to the outer sheath of the cable of Fig. 1. The computed conductor currents for different amplitudes of the driving current are normalized by dividing the values of the current with the amplitude of the driving current for each case.

The computed results in Fig. 5 are comparable with those shown in Fig. 6 [6]. The upper part of Figs. 5 and 6 is the impressed current in the sheath and the lower parts are currents induced in the central conductor for different intensities of the impressed current. This example of a thin ferromagnetic sheath conforms to the known conclusion that ferromagnetic materials are attractive from the viewpoint of high-frequency shielding [10]. However, saturation makes the ferromagnetic shields poorer than those of nonferromagnetic good conductors (such as copper or aluminum) due to substantially smaller conductivity of steel.

Nevertheless, Figs. 5 and 6 show that the shield provides good protection even against an impressed current of $1 \mathrm{kA}$. It appears
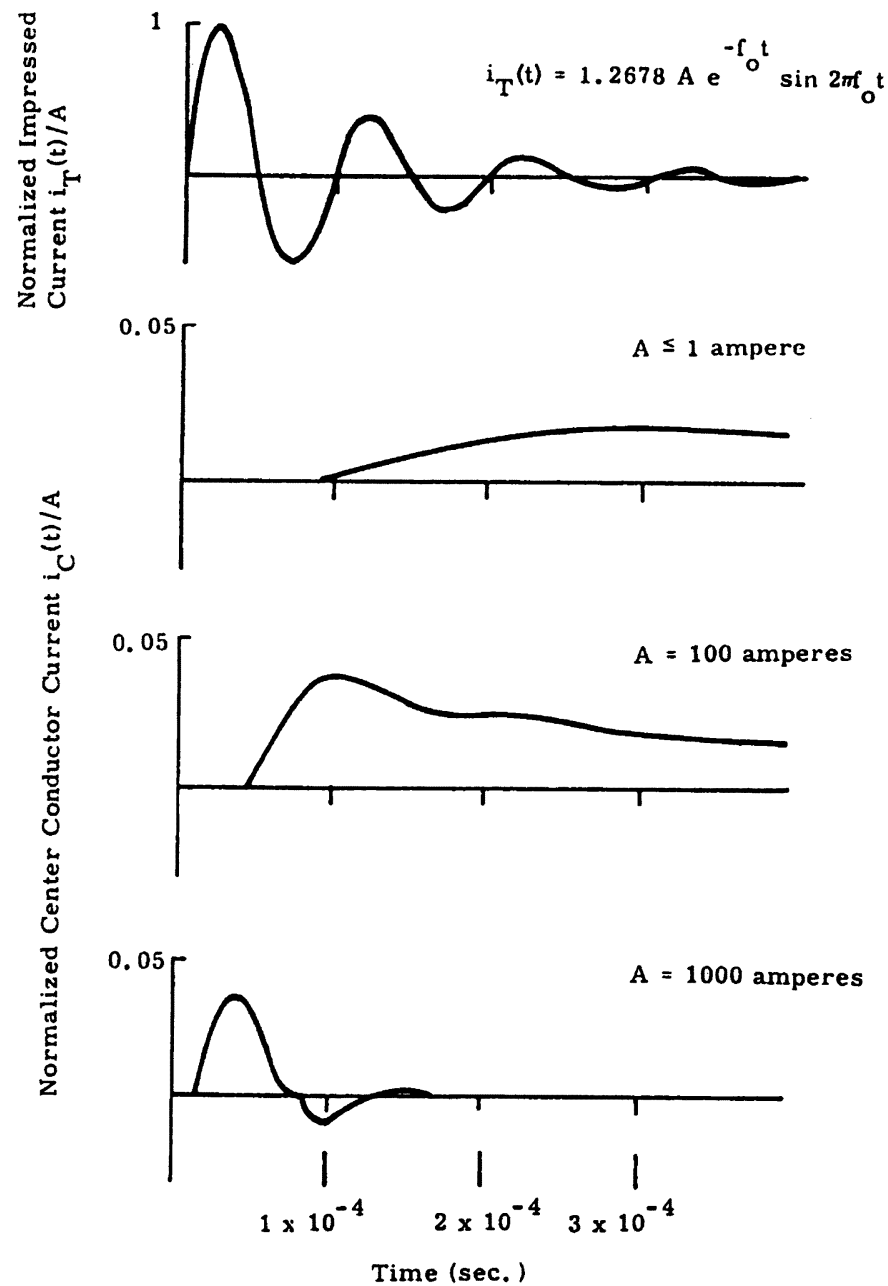

Fig. 6. Simulated normalized center conductor current for a damped sine wave of the driving current $i_{T} ; f_{0}=10 \mathrm{kHz}$ (saturation characteristic)—taken from [6].

that the current density in the inside layer and the electric field on the inside surface are small, and still good shielding is provided [10].

Fig. 7 shows the magnetic field variation in layers of the shield for $f_{0}=10 \mathrm{kHz}$ and a current amplitude of $1 \mathrm{kA}$ for the specific layers. The diminishing magnitude of the field for inner layers is attributed to the good shielding performance of the sheath for this frequency and current amplitude.

\section{B. Double-End Grounded Cable Sheath}

We consider a case where a cable connects two systems, similar to the case in [13] (Fig. 8). The cable sheath is connected to the grounding system at both ends and is in direct contact with the earth.

In this example, the length of the cable is $l=100 \mathrm{~m}$, the number of longitudinal sections is $n=25$ and the resistivity of the ground $\rho_{E}=100 \Omega \cdot \mathrm{m}$. The dimensions of the cable are the same as those shown in Table II, and the applied current is a damping wave expressed by (13) with an intensity of $500 \mathrm{~A}$ and a frequency of $10 \mathrm{kHz}$. The cross section is divided into ten layers. The sheath is grounded at both ends by a resistance of $R_{E 1}=R_{E 2}=20 \Omega$. Fig. 9 shows the computed normalized 


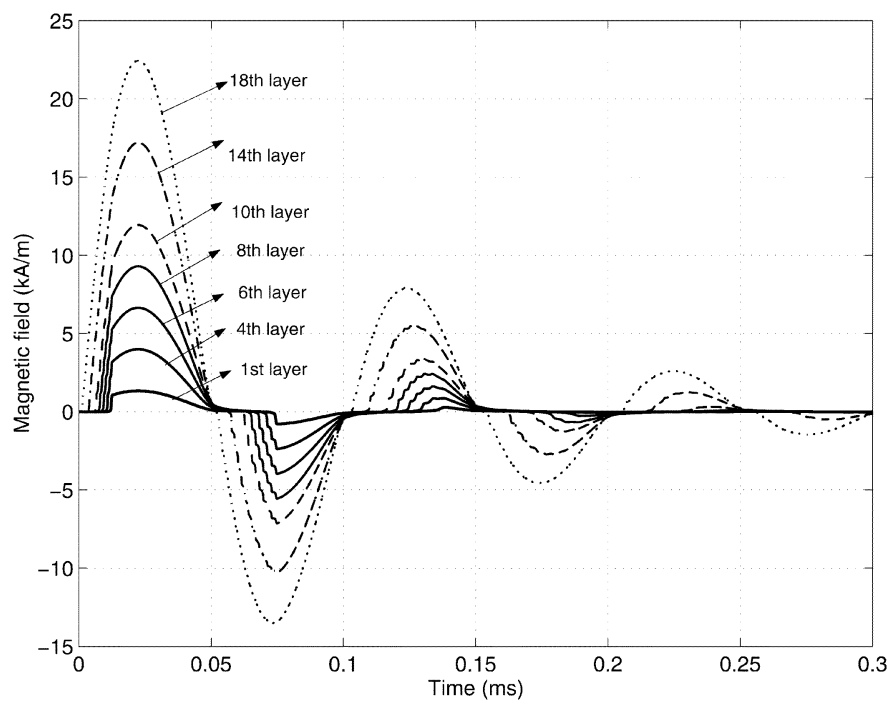

Fig. 7. Computed values of the magnetic field intensity for specific layers along the cross section of a cable sheath for a driving current $i_{T}$ with an amplitude of $1 \mathrm{kA}$ and frequency $f_{0}=10 \mathrm{kHz}$.

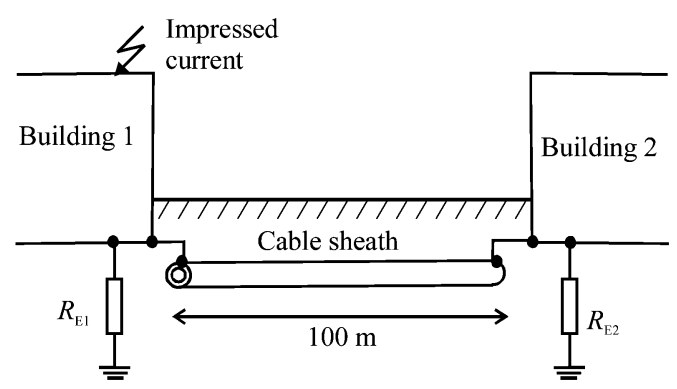

Fig. 8. Two systems connected by a cable sheath: partial impressed current is carried away by the cable sheath.

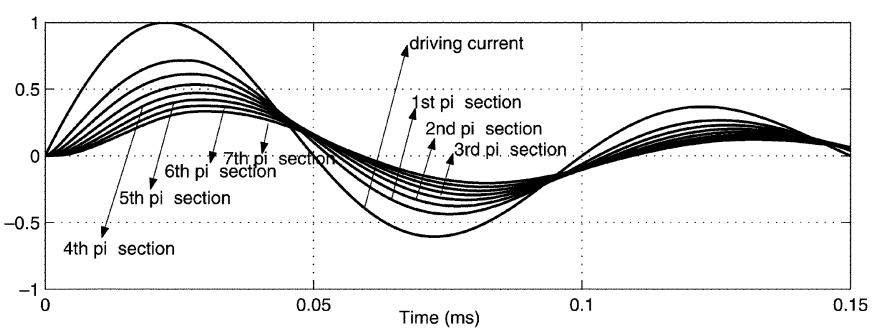

(a)

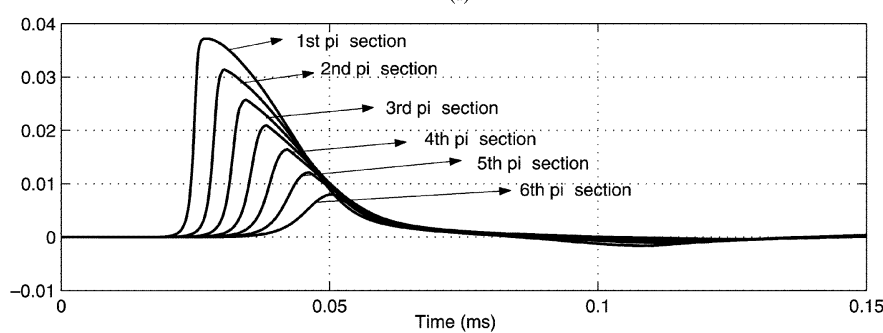

(b)

Fig. 9. Normalized current in segments along the cable (a) current in the outer layer of the sheath. (b) Induced current in a conductor inside the sheath.

currents on the outer layer and induced currents in the imaginary central conductor in SEGMENTS along the cable length.

In this example, the cable sheath carries away partial lightning or fault current in the first system. The cable sheath acts as a grounding electrode and dissipates part of the current. The

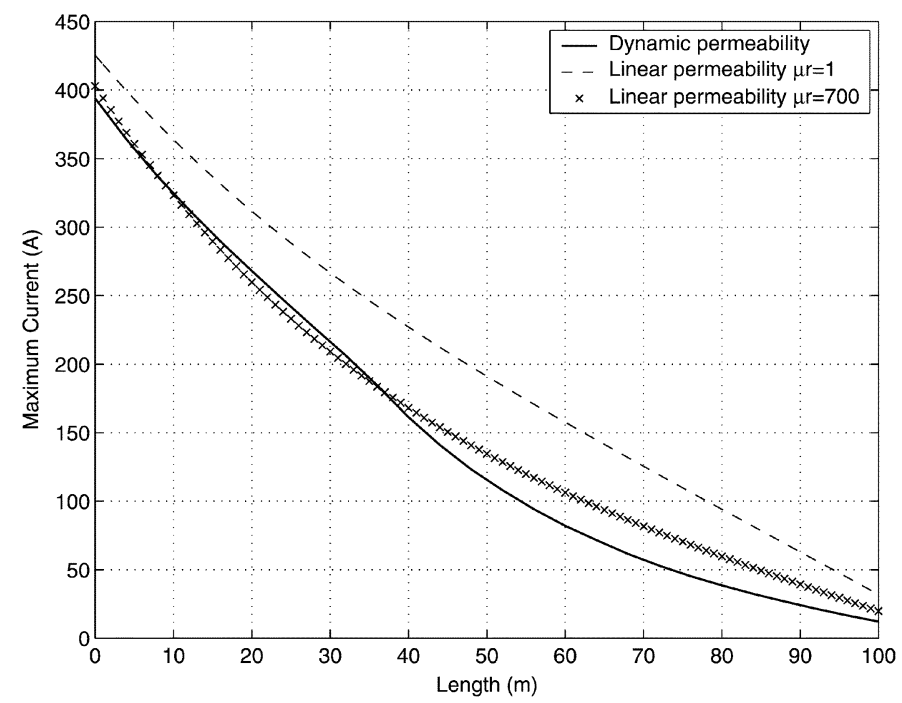

Fig. 10. Total current in the sheath along the cable: comparison between the ferromagnetic model and models with constant permeability with $\mu_{r}=700$ and $\mu_{r}=1$.

longitudinal currents in Fig. 9(a) diminish along the cable due to leakage currents. At the same time, the cable provides good shielding to the central conductors Fig. 9(b).

Figure 10 shows a comparison of the computed total current in the sheath along the cable length. Results by the proposed model are comparable with the transmission-line model [9] if linear inductivity of the sheath with relative permeability of 700 is assumed [10].

\section{CONCLUSION}

An approach for modeling the cable sheath dynamic behavior that takes into account its ferromagnetic properties has been presented. The developed model was implemented in ATP-EMTP using an equivalent circuit; the nonlinear inductances of which were determined from the EM field analysis in the time domain based on the Jiles model of hysteresis. The model simulates the complex dynamic behavior of the cable shield in conjunction with other parts of the electric power system. In this way, it allows one to study the shielding properties of the sheath in time domain in practical situations.

The ferromagnetic shields proved to have better shielding properties than good conductors that are commonly used, that is, when the magnetic material is not saturated. This is of special interest at higher frequencies where higher currents are required for complete saturation. It has been shown that a thin ferromagnetic sheath provides good shielding even when carrying 1-kA current at $10 \mathrm{kHz}$.

\section{REFERENCES}

[1] I. D. Mayergoyz, Mathematical Models of Hysteresis. New York: Springer, 1991.

[2] D. A. Philips, L. R. Dupre, and J. A. Melkebeek, "Comparison of Jiles and preisach hysteresis models in magnetodynamics," IEEE Trans. Magn., vol. 31, no. 6, pp. 3551-3553, Nov. 1995.

[3] D. C. Jiles and D. L. Atherton, "Theory of ferromagnetic hysteresis," $J$. Magn. Magn. Mater, vol. 61, pp. 48-60, 1986.

[4] D. C. Jiles et al., "Numerical determination of hysteresis parameters for modeling of magnetic properties using the theory of ferromagnetic hysteresis," IEEE Trans. Magn., vol. 28, no. 1, pp. 27-35, Jan. 1992. 
[5] "CIGRÉ Guide on Electromagnetic Compatibility in Electric Power Plants and Substations," CIGRÉ, Paris, France, Pub. 124, 1997.

[6] D. E. Merewether, "Analysis of the shielding characteristics of saturable ferromagnetic cable shields," IEEE Trans. Electromagn. Compat., vol. EMC-12, no. 3, pp. 134-137, Aug. 1970.

[7] L. Grcev, Z. Tasev, and L. Kocarev, "Shielding characteristics of hysteretic ferromagnetic tubular shields," in Proc. Int. Conf. Lightning Protection, Florence, Italy, 1996, pp. 685-689.

[8] R. Rudenberg, Electrical Shock Waves in Power Systems. Cambridge, MA: Harvard Univ. Press, 1968

[9] E. D. Sunde, Earth Conduction Effects in Transmission Systems. New York: Van Nostrand, 1949.

[10] E. F. Vance, Coupling to Shielded Cables. New York: Wiley, 1978.

[11] R. C. Dugan, R. G. Rocamora, and G. W. Brown, "Surge propagation in three-phase pipe-type cables, Part II-Duplication of field test including the effect of neutral wires and pipe saturation," IEEE Trans. Power App. Syst., vol. PAS-96, no. 3, pp. 826-833, May/Jun. 1977.

[12] J. Wiesinger, "Bestemmung der durch blitzströme in schirmrohren aus kupfer und aluminum entstehenden langsspannungen" (in German), ETZ Archiv, vol. H. 8, Bd. 2, pp. 239-242, 1980.

[13] A. Kern, J. Wiesinger, and W. Zischank, "Calculation of the longitudinal voltage along metal tubes caused by lightning currents and protection measures," in Proc. 7th Int. Symp. High Voltage Engineering, Dresden, Germany, 1991, pp. 147-150.

[14] Alternative Transients Program (ATP) Rule Book, Bonneville Power Administration (BPA), Canadian/American EMTP User Group, 1992.

[15] R. J. Meredith, "EMTP modeling of electromagnetic transients in multimode coaxial cables by finite sections," IEEE Trans. Power Del., vol. 12, no. 1, pp. 489-496, Jan. 1997.

[16] J. B. M. van Waes, "Safety and EMC aspects of grounding," Ph.D. thesis, Electr. Power Syst. Lab., Eindhoven Univ. Technol., Eindhoven, The Netherlands, Oct. 2003.

[17] L. Grcev, A. P. J. van Deursen, and J. B. M. van Waes, "Lightning current distribution to ground at power line tower with radio base station," IEEE Trans. Electromagn. Compat., vol. 47, no. 1, pp. 160-170, Feb. 2005.

[18] R. G. Olsen and M. C. Willis, "A comparison of exact and quasistatic methods for evaluating grounding systems at high frequencies," IEEE Trans. Power Del., vol. 11, no. 2, pp. 1071-1081, Apr. 1996.

[19] M. Heimbach and L. Grcev, "Grounding system analysis in transients programs applying electromagnetic field approach," IEEE Trans. Power Del., vol. 12, no. 1, pp. 186-193, Jan. 1997.

[20] S. Prigozy, "PSPICE computer modeling of hysteresis effects," IEEE Trans. Educ., vol. 36, no. 1, pp. 2-5, Feb. 1993.

[21] L. Grcev, Z. Tasev, and L. Kocarev, "Frequency dependent model of ferromagnetic hysteresis for time domain analysis of cable shields," in Proc. IEEE Int. Symp. Electromagnetic Compatibility, Austin, TX, 1997, pp. 586-591.

[22] L. Grcev and V. Arnautovski, "Lightning currents coupling to metal tubes," in Proc. Int. Telecommunications Energy Conf., Paris, France, 1993, Paper 28.B.3, pp. 187-192.

[23] F. Menter and L. Grcev, "EMTP-Based model for grounding systems analysis," IEEE Trans. Power Del., vol. 9, no. 4, pp. 1838-1849, Oct. 1994.

[24] C.-S. Yen, Z. Fazarinc, and R. L. Wheeler, "Time-domain skin-effect model for transient analysis of lossy transmission lines," Proc. IEEE, vol. 70, pp. 750-757, 1982

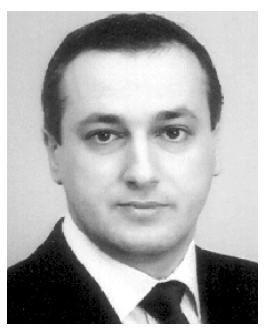

Marjan Popov (M'95-SM'03) was born in Kavadarci, Macedonia, in 1969. He received the Dipl.-Ing. and M.S. degrees in electrical engineering from the Saints Cyril and Methodius University, Skopje, Macedonia, in 1993 and 1998, respectively, and the Ph.D. degree from Delft University of Technology (TU), Delft, The Netherlands, in 2002.

Currently, he is an Assistant Professor with the group of Electrical Power Systems, Power Systems Laboratory, TU Delft. From 1993 to 1998, he was a Teaching and Research Assistant with the Faculty of Electrical Engineering, University of Skopje. In 1997, he was an Academic Visitor with the University of Liverpool, Liverpool, U.K. His fields of interest are arc modeling, transients in power systems, parameter estimation, and relay protection.

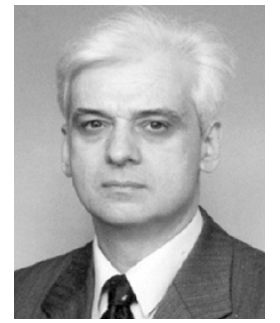

Leonid Grcev (M'84-SM'97) was born in Skopje, Macedonia, in 1951. He received the Dipl.-Ing. degree in electrical engineering from the Saints Cyril and Methodius University, Skopje, in 1978, and the M.S. and Ph.D. degrees in electrical engineering from the University of Zagreb, Zagreb, Croatia, in 1982 and 1986, respectively.

Currently, he is a Full Professor with the Faculty of Electrical Engineering at the Saints Cyril and Methodius University, where he has also been Assistant Professor, Associate Professor, and Vice Dean since 1988. From 1978 to 1988, he was with the Electric Power Company of Macedonia, Skopje, working in the Telecommunications Department. He has been a Visiting Professor at the Technical University of Aachen, Aachen, Germany, the Eindhoven University of Technology, Eindhoven, The Netherlands; and the Swiss Federal Institute of Technology, Lausanne, Switzerland. He was responsible for several international projects related to EMC. His research interests include EMC of power systems and modeling of grounding systems and connected structures at high frequencies and transients with particular reference to lightning. He is author or coauthor of many scientific papers published in reviewed journals and presented at international conferences.

Dr. Grcev is a member of the CIGRE Working Group C4.04 (General Aspects of EMC and EMF) and has been Chairperson and a member of scientific committees at international conferences.

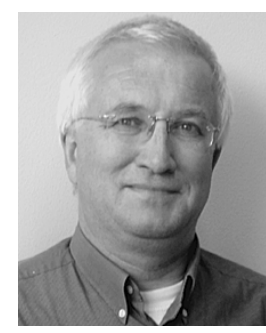

Lou van der Sluis (SM'86) was born in Geervliet, The Netherlands, on July 10, 1950. He received the M.Sc. degree in electrical engineering from the Delft University of Technology (TU), Delft, The Netherlands, in 1974.

Currently, he is a Full-Time Professor in the Power Systems Department, TU Delft, where he was a PartTime Professor in 1990. In 1977, he became a Test Engineer with the KEMA High Power Laboratory, Arnhem, The Netherlands, and was involved in the development of a data-acquisition (DAQ) system for the High Power Laboratory, computer calculations of test circuits, and the analysis of test data by digital computer.

Prof. van der Sluis is Past Chairman of CC-03 of CIGRE and CIRED to study the transient recovery voltages in medium- and high-voltage networks.

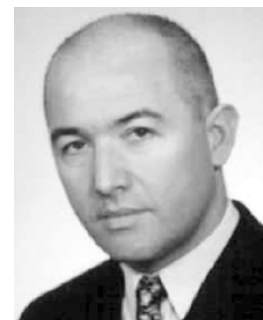

Vladimir V. Terzija (M'95-SM'00) was born in Donji Baraći, Bosnia and Herzegovina, in 1962. He received the B.Sc., M.Sc., and Ph.D. degrees in electrical power engineering from the Department of Electrical Engineering, University of Belgrade, Serbien and Montenegro, Yugoslavia, in 1988, 1993 and 1997, respectively.

Currently, he is an expert on protection, control, and monitoring of medium-voltage switchgears with ABB Calor Emag Mittelspannung, Ratingen, Germany, where he has been since 2001. In 1988, he was an Assistant Professor at the University of Belgrade, teaching courses in electric power quality, power system control, electromechanic transient processes in power systems, and estimation techniques in power engineering.

Dr. Terzija became a Research Fellow at the Institute of Power Engineering, Saarland University, Saarbruecken, Germany, in 2000, granted by the Alexander von Humboldt Foundation. 